\title{
1 Increase in phospholamban content in mouse skeletal muscle after denervation
} 2

3 Masatoshi Komatsu ${ }^{1,2}$, Tsutomu Nakada ${ }^{1,3 *}$, Hiroyuki Kawagishi ${ }^{1,4}$, Hiroyuki Kato ${ }^{2}$, and Mitsuhiko 4 Yamada $^{1 *}$.

5

$6{ }^{1}$ Department of Molecular Pharmacology, Shinshu University School of Medicine, Matsumoto, Nagano,

7 Japan, ${ }^{2}$ Department of Orthopaedic Surgery, Shinshu University School of Medicine, Matsumoto,

8 Nagano, Japan, ${ }^{3}$ Department of Instrument Analysis, Research Center for Supports to Advanced Science,

9 Shinshu University, Matsumoto, Nagano, Japan. ${ }^{4}$ Department of Biotechnology and Biomedical

10 Engineering, Institute for Biomedical Science, Shinshu University, Matsumoto, Nagano, Japan.

12 Corresponding to:

13 Tsutomu Nakada, Ph.D. (ORICD: 0000-0002-7662-6742)

14 Department of Instrument Analysis,

15 Research Center for Supports to Advanced Science,

16 Shinshu University

17 3-1-1 Asahi, Matsumoto, Nagano 390-8621 Japan.

18 FAX: $+81-263-37-3085$

19 TEL: $+81-263-37-2606$

20 E-mail: tnakada@shinshu-u.ac.jp

21 or

22 Mitsuhiko Yamada, MD, Ph.D. (ORICD: 0000-0002-7515-3824)

23 Department of Molecular Pharmacology,

24 Shinshu University School of Medicine

25 3-1-1 Asahi, Matsumoto, Nagano 390-8621 Japan.

26 FAX: $+81-263-37-3085$

27 TEL: $+81-263-37-2605$

28 E-mail:myamada@shinshu-u.ac.jp 
It is well-known that denervation of motor nerves induces atrophy and decreases contractile

4 force of the skeletal muscle. However, it is not completely understood how denervation alters calcium

5 handling in the skeletal muscle. We investigated the effect of denervation on the expression and function

6 of proteins involved in calcium handling. Two weeks after denervation of the right sciatic nerve in mice,

7 we observed a significant decrease in mass and cross-sectional area of the ipsilateral tibialis anterior (TA)

8 and flexor digitorum brevis (FDB) muscles. Also, we observed a significant decrease in the specific

9 tetanus contractile force in the ipsilateral TA muscle. Calcium imaging of the ipsilateral FDB showed that

10 the peak twitch and tetanus calcium concentrations were significantly decreased due to a decrease in

11 calcium content of the sarcoplasmic reticulum (SR). Denervation reduced the maximum rate of

12 sarcoplasmic/endoplasmic calcium ATPase (SERCA) activity. Interestingly, the amount of

13 phospholamban (PLN), but not its transcripts, was increased in the ipsilateral vs. contralateral side after

14 denervation, suggesting that denervation impairs constitutive regulation of PLN. Immunohistochemical

15 analysis revealed increased PLN in all major fiber types in TA with IIx fibers showing a threefold higher

16 expression than the contralateral side. These results suggest that the abnormal increase in PLN in the

17 ipsilateral fast-twitch fibers may be involved in decreased SERCA activity, SR calcium content, peak

18 calcium transients, and contractile forces of denervated muscles.

20 Keywords: denervation/skeletal muscle/sarcoplasmic/endoplasmic calcium ATPase/phospholamban/ 
Calcium cycling between the sarcoplasmic reticulum (SR) and cytosol is a fundamental mechanism of skeletal muscle contraction. In skeletal muscle fibers, action potential-induced membrane depolarization is converted to calcium release from the SR by the cooperative action of $\mathrm{Ca}_{\mathrm{V}} 1.1 \mathrm{~L}$-type calcium channels in T-tubules and ryanodine receptors (RyR1) on the SR (Allard 2018). Released calcium is then reuptaken into the SR by sarcoplasmic/endoplasmic reticulum calcium ATPase (SERCA) (Brini and Carafoli 2009; Allard 2018). Activities of these channels and pumps are tightly regulated by several mechanisms, such as phosphorylation and protein-protein interactions. Impairment of these regulatory mechanisms would lead to catastrophic impairment of muscle contraction.

Sciatic nerve denervation is one of the most popular experimental models of skeletal muscle atrophy (Midrio 2006). This model simulates the clinical outcome of various motor neuron diseases or injuries and may also simulate a part of pathophysiology of the age-induced sarcopenia known to be associated with morphological and functional changes in neuromuscular junctions (Gonzalez-Freire et al. 2014; Rudolf et al. 2014; Park 2015). Denervation causes acute muscle atrophy and a decreased contractile force through different physiological, histological, and biochemical changes, depending on denervation periods, muscle types, and animal species. Several reports showed that denervation alters the expressions and activities of various proteins involved in calcium homeostasis in the skeletal muscle (Dufresne et al. 2016; Loirat et al. 1988; Salvatori et al. 1988; Schulte et al. 1994; Péréon et al. 1997).

Skeletal muscles can be largely classified into two groups; slow-twitch muscles that are composed of type I fibers (slow, oxidative, and fatigue resistant), and fast-twitch muscles that are mainly composed of type IIa (fast, oxidative, fatigue resistant), IIx (fast, glycolytic, and fatigue-intermediate), and IIb (fast, glycolytic, and fatigable) fibers (Schiaffino and Reggiani 2011). In addition, there are hybrid fibers that exhibit an intermediate phenotype of distinct fiber types (Schiaffino and Reggiani 2011). In mice, the soleus is the prototypical slow-twitch muscle, whereas the flexor digitorum brevis (FDB), extensor digitorum longus, and tibialis anterior (TA) are representative of fast-twitch muscles. These fiber types show different physiological, structural, and biological properties (Schiaffino and Reggiani 2011).

In particular, expression of SERCA isoforms and their associated proteins are distinctly different between the slow- and fast-twitch fibers (Periasamy and Kalyanasundaram 2007). SERCA is a member of the ATP-driven calcium pump family which uptake calcium from the cytosol into the SR in the striated muscles. Three genes of SERCA family (SERCA1-3) have been identified in the mouse genome, and several splice variants are produced from each gene (Brini and Carafoli 2009). In the skeletal and cardiac muscles, SERCA activity is regulated by small transmembrane proteins such as phospholamban (PLN) and sarcolipin (SLN). PLN and SLN directly interact with SERCA and thus negatively regulate calcium transport activity. In cardiac and slow-twitch muscle, SERCA2 and PLN are dominantly expressed, whereas, in the fast-twitch muscles, SERCA1 and SLN are abundant (Periasamy and Kalyanasundaram 2007; Fajardo et al. 2013). Previous reports indicate that denervation causes 
1 SERCA isoforms is changed (Dufresne et al. 2016; Schulte et al. 1994). However, the underlying 2 mechanism of SERCA activity suppression has not been identified unequivocally.

3 In this study, we demonstrate that expression of PLN was dramatically increased, and

4 SERCA activity halved in the ipsilateral TA muscle 2 weeks after right sciatic nerve transection in mice.

5 The amount of PLN expression was increased in all fast fiber types, especially IIx fibers in the ipsilateral

6 TA muscle. Furthermore, we found that the twitch and tetanus calcium transients were smaller due to the

7 smaller SR content in the ipsilateral compared with contra-lateral FDB. These results suggest that

8 abnormal PLN in the fast-twitch muscle fibers may be involved in decreased SERCA activity and

9 contractile forces of denervated skeletal muscles.

10 
Animals

All experimental procedures were conducted in accordance with the Guidelines for Animal Experimentation of Shinshu University and approved by the Committee for Animal Experimentation. Isolation of skeletal muscles was performed in ten 13-week-old male C57BL/6 mice. All mice were given free access to water and standard diet throughout the study and were maintained in a temperature-controlled $\left(21{ }^{\circ} \mathrm{C}-26^{\circ} \mathrm{C}\right)$ and humidity-controlled $(50 \%-60 \%)$ room with a $12 \mathrm{~h}$ light/dark cycle. All experimental mice were anesthetized deeply by intraperitoneal administration of $0.3 \mathrm{mg} / \mathrm{kg}$ medetomidine (Domitor, Nippon Zenyaku Kogyo Co., Fukushima, Japan), 4.0 mg/kg midazolam (Midazolam Sandoz, Novartis, Tokyo, Japan), and $5.0 \mathrm{mg} / \mathrm{kg}$ butorphanol (Vetorphale, Meiji Seika Pharma Co., Tokyo, Japan). The right hindlimb muscles were denervated by excising a 3-4 mm segment of ipsilateral sciatic nerve.

\section{Measurement of contractile forces}

The contractile forces of TA muscles were measured in vivo according to the methods of a previous study with minor modifications (Cosgrove et al. 2014). The anesthetized mice were placed into $50 \mathrm{~mL}$ conical tubes with their hind limbs extending out through two holes. An incision was made in the skin above the TA muscle; then the distal tendon was sutured to a force transducer (Nihon Kohden, Tokyo, Japan). The muscle was stimulated with needle electrodes placed on both sides of the TA. The TA muscles were stretched to a resting tension of $100 \mathrm{mN}$ and electrically stimulated with $1 \mathrm{~ms}$ pulses of predetermined supramaximal voltage at $1 \mathrm{~Hz}$ (twitch) and $100 \mathrm{~Hz}$ (tetanus). Data were recorded using PowerLab 4/30 (AD Instruments, Dunedin, New Zealand). Contractile force, contraction rate ( $+\mathrm{dF} / \mathrm{dt})$, and relaxation rate $(-\mathrm{dF} / \mathrm{dt})$ were calculated by LabChart software (AD instruments). After the force measurement, TA muscles were fixed overnight with $10 \%$ neutral buffered formalin at $4{ }^{\circ} \mathrm{C}$ and used for conventional hematoxylin and eosin (HE) staining. The cross-sectional area was calculated from the specimens using ImageJ. The specific force was calculated by normalizing the contractile force to the cross-sectional area.

\section{FDB fibers isolation and calcium imaging}

FDB fibers were dissected and digested in Dulbecco's Modified Eagle Medium (DMEM) containing $5 \mathrm{mg} / \mathrm{ml}$ collagenase type 2 (Worthington Biomedical, Lakewood, $\mathrm{NJ}$ ) at $37^{\circ} \mathrm{C}$ with agitation for $1 \mathrm{~h}$. After digestion, the fibers were washed four times with DMEM containing 10\% FBS to eliminate the collagenase. Isolated FDB fibers were then plated onto laminin-coated coverslips. After 30 min of incubation at $37^{\circ} \mathrm{C}$, the fibers were incubated with $5 \mu \mathrm{M}$ Fluo-4/AM (Dojindo, Kumamoto, Japan) plus $0.01 \%$ Cremophor EL (Sigma-Aldrich, St. Louis, MO) and $0.02 \%$ bovine serum albumin (Sigma-Aldrich) in serum-free DMEM for $45 \mathrm{~min}$ at $37^{\circ} \mathrm{C}$, followed by de-esterification. The dye-loaded cells were then superfused with modified Tyrode solution (136.5 mM NaCl, $5.4 \mathrm{mM} \mathrm{KCl,} 1.8 \mathrm{mM} \mathrm{CaCl}_{2}$, 
$0.53 \mathrm{mM} \mathrm{MgCl}_{2}, 5.5 \mathrm{mM}$ HEPES, and $5.5 \mathrm{mM}$ glucose, $\mathrm{pH}$ 7.4) at room temperature and paced with 1 ms pulses of $50 \mathrm{~V}$ at $0.3 \mathrm{~Hz}$ (twitch) or $1 \mathrm{~ms}$ pulses of $50 \mathrm{~V}$ at $50 \mathrm{~Hz}$ (tetanus) across the $20 \mathrm{~mm}$ incubation chamber. Fluorescent images were acquired with an LSM 7 LIVE laser scanning microscope and $20 \times / 0.8$ plan apochromatic objective (Carl Zeiss, Oberkochen, Germany). Fluo- 4 was excited by $488 \mathrm{~nm}$ light, and the emission light was passed through a high-pass filter of $495 \mathrm{~nm}$ and captured by a charge-coupled device $(\mathrm{CCD})$ camera. Image resolution of $128 \times 128$ pixels was acquired every $2.8 \mathrm{~ms}$. The time course of calcium transients was obtained from the fluorescence change in individual cells selected by a region of interest (ROI) tool. The time-to-peak and the half decay time (T1/2) were measured with an in-house computer program written with LabVIEW ${ }^{\circledR}$ (National Instruments Japan Corp., Tokyo, Japan). To estimate SR calcium content, fibers were exposed to a calcium release cocktail (ICE) containing $10 \mu \mathrm{M}$ ionomycin, $30 \mu \mathrm{M}$ cyclopiazonic acid (CPA), and $100 \mu \mathrm{M}$ EGTA in calcium-free

\section{Preparation of microsomes}

The TA muscles were dissected from the mice and ground in liquid nitrogen with a mortar and pestle. The powdered samples were homogenized in homogenization buffer (320 mM sucrose, 20 mM HEPES, pH 7.4) containing a protease inhibitor cocktail (Roche Diagnostics, Rotkreuz, Switzerland) using a grass Teflon homogenizer (Sansyo, Tokyo, Japan), and then centrifuged at 2,000 $\times \mathrm{g}$ for $15 \mathrm{~min}$ at $4{ }^{\circ} \mathrm{C}$ to remove any cell debris. The supernatants were ultracentrifuged at $100,000 \times \mathrm{g}$ for $60 \mathrm{~min}$ at $4{ }^{\circ} \mathrm{C}$. For measurement of SERCA activity, the resultant pellets were resuspended with homogenization buffer and stored at $-80{ }^{\circ} \mathrm{C}$ until use. For western blotting, the resultant pellets were dissolved with lysis buffer (10 mM Tris, 150 mM NaCl, 5 mM EDTA, $1 \%$ Triton X-100, 10\% glycerol, $\mathrm{pH}$ 7.4) containing protease inhibitor cocktail and incubated at $4{ }^{\circ} \mathrm{C}$ on a rotator for $1 \mathrm{~h}$. The lysates were then centrifuged at $8,000 \times$ g for $30 \mathrm{~min}$ at $4{ }^{\circ} \mathrm{C}$ to remove insoluble materials and stored at $-80{ }^{\circ} \mathrm{C}$ until use. Protein concentrations were measured with a BCA protein assay kit (Thermo Fisher Scientific, Waltham, MA).

\section{Measurement of SERCA activity}

SERCA activity was determined as the production of inorganic phosphate by colorimetric method according to a previous study with minor modifications (Duhamel et al. 2007). Briefly, $0.2 \mu \mathrm{g}$ of TA microsomes were added to $50 \mu 1$ of uptake buffer ( $20 \mathrm{mM}$ MOPS, $100 \mathrm{mM} \mathrm{KCl}, 5 \mathrm{mM} \mathrm{MgCl}, 5 \mathrm{mM}$ K-oxalate, $5 \mathrm{mM} \mathrm{NaN}_{3}, 0.5 \mathrm{mM}$ EGTA, $1 \mathrm{mM} \mathrm{Na}_{2}$-ATP, pH 7.45 with $\mathrm{KOH}$ ) with various $\mathrm{CaCl}_{2}$ concentrations. After a $30 \mathrm{~min}$ incubation period at $37^{\circ} \mathrm{C}$ with or without $\mathrm{CPA}$, the reaction was stopped by adding EGTA to final concentration $100 \mathrm{mM}$. Concentration of inorganic phosphate in the resulting mixture was determined by BIOMOL ${ }^{\circledR}$ Green Reagent (Enzo Life Science, Farmingdale, NY) according to the manufacturer's instructions. The SERCA activity was calculated as the difference between CPA-sensitive and CPA-insensitive inorganic phosphate production. The data were fitted using the 
3 where $y$ is SERCA activity; $V_{\max }$, the maximum SERCA activity; $K_{1 / 2}$, the half-maximum calcium

4 concentration; $x$, the calcium concentration; and $h$, the Hill coefficient. In the results section, $K_{1 / 2}$ is

5 expressed as its negative logarithm $\left(\mathrm{pCa}_{50}\right)$.

\section{Western blotting} electrophoresis (SDS-PAGE) and electroblotted onto polyvinylidene difluoride membranes. The membranes were blocked with 5\% nonfat skim milk in TBST (150 mM NaCl, $10 \mathrm{mM}$ Tris, and $0.1 \%$ Tween 20, $\mathrm{pH}$ 7.4) for $1 \mathrm{~h}$ at room temperature and then incubated at $4{ }^{\circ} \mathrm{C}$ with primary antibodies overnight. The primary antibodies used were anti-SERCA1 (mouse monoclonal, clone VE121G9, Abcam, Cambridge, UK), anti-SERCA2 (mouse monoclonal, clone 2A7-A1, Thermo Fisher Scientific), anti-STIM1 (mouse monoclonal, ab57834, Abcam), anti-Orai1 (rabbit polyclonal, AB9868, Merck Millipore), anti-Ca 1.1 (mouse monoclonal, ab2862, Abcam), anti-RyR (mouse monoclonal, clone 34C, Thermo Fisher Scientific), anti-parvalbumin (rabbit polyclonal, HPA048536, Sigma-Aldrich), anti-PLN (mouse monoclonal, clone 2D12, Abcam), and anti-PLN (rabbit polyclonal, HPA026900, Sigma-Aldrich). After washing with TBST, membranes were incubated in horseradish peroxidase (HRP)-conjugated donkey anti-rabbit IgG or HRP-conjugated donkey anti-mouse IgG (Jackson ImmunoResearch Laboratories, West Grove, PA) for $1 \mathrm{~h}$ at room temperature. The bound secondary antibody was visualized with Immobilon Western (Millipore, Billerica, MA) according to the manufacturer's instructions.

\section{QPCR} Japan) according to the manufacturer's instructions. One microgram of total RNA was used as a template for reverse transcription using SuperScript III First-Strand synthesis system for qRT-PCR (Invitrogen, Carlsbad, CA). For real-time PCR, FastStart Universal SYBR Green Master (Rox) (Roche Diagnostics) was performed according to the manufacturer's instructions. For calculating data, $\Delta \Delta \mathrm{CT}$ method with internal control as glyceraldehyde-3-phosphate dehydrogenase (GAPDH) was used. The primers used were 5'-TGCCTCATCTTGATATGTCTG-3' (PLN, sense), 5'-CACTTAGCCACTGTAAAGTTTGT-3' (PLN, antisense), 5'-TCATGACCACAGTCCATGCCATC-3' (GAPDH, sense), and 5'-AGGTCCACCACCCTGTTGCT-3' (GAPDH, antisense). Real-time PCR was performed with a StepOnePlus PCR device (Thermo Fisher Scientific).

\section{Immunohistochemistry}


1 cryostat and immunostained without fixation. Briefly, after washing with PBS, specimens were blocked 2 and permeabilized with PBS containing $0.2 \%$ Triton and 5\% FBS for $1 \mathrm{~h}$ at room temperature and then 3 incubated with the indicated primary antibodies at $4{ }^{\circ} \mathrm{C}$ overnight. After washing with PBS, cells were 4 then incubated with fluorescent dye-conjugated secondary antibodies and Hoechst 33342 (Dojindo) at 5 room temperature for $1 \mathrm{~h}$. Cells were again washed with PBS, and coverslips were mounted with 6 Fluoromount-G (Beckman Coulter, Fullerton, CA). For fiber typing, anti-MHCI (BA-F8), anti-MHCIIa 7 (SC-71), anti-MHCIIb (BF-F3), and anti-MHCIIx (6H1) antibodies (Developmental Studies Hybridoma 8 Bank) were used as previously described (Bloemberg and Quadrilatero 2012). Rabbit anti-PLN-antibody 9 (HPA026900, Sigma-Aldrich) was used to stain the PLN. To visualize the signals, Alexa Fluor 350 goat 10 anti-mouse $\operatorname{IgG}_{2 \mathrm{~b}}$, Alexa Fluor 488 goat anti-mouse $\mathrm{IgG}_{1}$, Alexa Fluor 555 goat anti-mouse IgM, Alexa 11 Fluor 555 donkey anti-rabbit IgG (all from Invitrogen) were used as secondary antibodies. Fluorescent 12 images were acquired with a fluorescent microscope AxioObserverZ1 (Carl Zeiss). Fluorescent intensity 13 and cross-sectional area of each fiber were calculated by ImageJ with an ROI tool.

\section{Statistical analysis}

All results are expressed as mean \pm standard error (SE). Student's unpaired $t$-test was used to 17 examine differences between two means of data. $P<0.05$ was considered to be significant. 
3 Decrease in absolute and specific contractile forces after denervation

We first investigated the effect of the right sciatic nerve denervation on the contractile force and cross-sectional area of both ipsi- and contra-lateral TA muscles. On the $14^{\text {th }}$ day of denervation, both the mass and cross-sectional area of the ipsilateral TA muscle were significantly decreased compared with the contralateral muscles (Fig. 1a). Contractile forces (elicited by twitch- and tetanic-stimulations) were also dramatically decreased in ipsi- vs. contra-lateral TA muscle after the denervation (Fig. 1b). Interestingly, the specific tetanus, but not twitch force, of ipsilateral TA muscle was significantly decreased compared with the contralateral muscle (Fig. 1c). Relaxation rate (-dF/dt) was also significantly decreased in tetanus but not twitch. These results suggested that denervation altered the response of the TA muscle to high-frequency stimulations possibly by reducing the activity of SERCA, a critical enzyme determining calcium homeostasis in the skeletal muscle.

\section{Changes in calcium transient in single muscle fiber after denervation}

Thus, we performed calcium imaging by using a fluorescent calcium indicator Fluo-4. Since it is difficult to isolate a single fiber from the TA muscle, we used isolated FDB fibers in these analyses. The maximum amplitudes of both twitch and tetanus calcium transients were significantly decreased in the ipsilateral side after denervation (Figs. $2 \mathrm{a}$ and $\mathrm{b}$ ). Time to peak and half decay time (T1/2) of calcium transient elicited by twitch stimulation were also significantly increased after denervation (Fig. 2a). We also applied a calcium releasing cocktail (ICE) containing ionomycin, CPA, and EGTA to determine the SR calcium content of the fiber. ICE application showed that the SR calcium content of the denervated fibers was significantly reduced compared with contralateral fibers (Fig. 2c). These results suggested that a reduction in the SR calcium content due to the decreased activity of SERCA might attenuate twitch and tetanus calcium transients.

\section{Reduction of SERCA activity in denervated fibers}

In order to confirm if SERCA activity is also compromised in the denervated TA muscle, we directly assayed its SERCA activity. The SERCA activity of the denervated TA muscle was significantly reduced at pCa of 5.0, 5.5, and 6.0 compared with that of the contralateral muscle (Fig. 3a). Fitting of these concentration-activity relationships into Eq. 1 revealed that $\mathrm{pCa}_{50}$ was unchanged, whereas $V_{\max }$ was significantly decreased after denervation (Fig. 3b). This result suggested that a decline in the SERCA activity might underlie the reduced force generation of the TA muscle (Fig. 1).

\section{Changes in expressions of proteins related to calcium homeostasis}

To investigate the causes of decline in SERCA activity, we compared expressions of proteins involved in calcium homeostasis between denervated and intact TA muscles. Western blotting showed that SERCA1 expression was significantly decreased after denervation $(22.6 \pm 5.8 \%$ reduction. $n=4)$ (Figs. 
$14 \mathrm{a}$ and $\mathrm{b}$ ). On the other hand, SERCA2 expression was significantly increased by denervation (Figs. 4a,

2 and b). However, the $\sim 40 \%$ reduction of SERCA activity could not be accounted for by these changes

3 alone. Therefore, we further analyzed expression of phospholamban (PLN), which is negative regulators

4 of SERCA activity. PLN dramatically increased in ipsilateral but not contra-lateral TA muscles (Fig. 4c).

5 Under the current experimental condition, only the monomer ( $6 \mathrm{kDa})$, but not the pentamer, of PLN was

6 observed. Western blotting with another antibody against PLN also confirmed a similarly substantial

7 increase in PLN (Fig. 4c). PLN also increased in the ipsilateral soleus muscles (Fig. 5a). However,

8 real-time PCR showed that the amount of PLN mRNA was not changed after denervation in either

9 ipsilateral TA or soleus muscles (Figs. 4d and 5b), suggesting that the increase in PLN protein resulted

10 from posttranslational alterations. Expression of other SR proteins such as STIM1 and calsequestrin 2

11 (CSQ2) were also significantly increased, but that of other proteins involved in calcium homeostasis

12 (CSQ1, parvalbumin, Orai1, $\mathrm{Ca}_{\mathrm{V}} 1.1$, and RyR1) was not significantly changed after denervation (Figs. 4a

13 and, b). These results suggest that the increase in PLN proteins may decrease SERCA activity in

14 denervated muscles.

\section{PLN expression in each fiber type}

17 The TA muscle is composed of several types of muscle fiber (I, IIa, IIx, IIb, and hybrids). We 18 investigated in which fiber types PLN was increased by using immunohistochemistry. The TA muscle 19 was analyzed separately in red (RTA) and white (WTA) portions. Fiber types of serial sections were 20 identified with immunohistochemistry with antibodies against each myosin heavy chain (MHC). There were few Type I fibers in TA muscle of both sides as previously reported (Bloemberg and Quadrilatero

22 2012). On the $14^{\text {th }}$ day of denervation, there was no significant change in fiber distribution, except for a 23 slight but significant increase in the type IIx fibers in RTA (Fig. 6a). We also investigated changes in 24 CSA of major fiber types (IIa, IIx, and IIb) in RTA (Fig. 6a). Denervation strongly reduced the CSA of 25 IIx (27.4 $\pm 3.1 \%$ reduction) and IIb (56.8 $\pm 2.0 \%$ reduction) but not IIa (3.5 $\pm 3.8 \%$ reduction) fibers. 26 PLN was increased in all the major types of fibers with Type IIx fiber showing as much as the threefold greater PLN amount in ipsi- than contra-lateral muscles (Fig. 6b). 
In this study, for the first time, we demonstrate that denervation increased the amount of PLN, thereby inhibiting SERCA activity in the fast-twitch muscle and thus reducing SR calcium content. We measured the contractile force, SERCA activity, and protein expression in the TA muscle but the calcium homeostasis in the FDB muscle. However, these distinct sets of the data seem to be consistent with each other because both the decrease in the relaxation rate of the TA muscle (Fig. 1c) and the increase in T1/2 of calcium transient (Fig. 2a) indicate the impairment of SERCA activity after denervation. Thus, we concluded that the reduced SR calcium content due to impairment of SERCA suppressed a peak calcium transient and decreased specific tetanic force in the fast-twitch fibers.

It is believed that PLN is expressed only in the cardiac muscle and slow-twitch type muscles (Jorgensen and Jones 1986). However, the present study demonstrates that denervation markedly increased the amount of PLN in both ipsilateral fast and slow muscles. Thus, it is likely that the amount of PLN in the skeletal muscle is constitutively regulated by the motor nerve under physiological conditions. It is noteworthy that this upregulation was a posttranslational phenomenon (Fig. 4d). Several reports indicate that cardiac PLN is degraded by proteasome or autophagy pathways in a ubiquitin-dependent manner (Teng et al. 2015; Nakagawa et al. 2016; Yokoe and Asahi 2017). Therefore, denervation may suppress the constitutive motor nerve-derived ubiquitination of PLN by mechanism such as down-regulation of E3 ubiquitin ligase specific to PLN. It is also possible that the increased expression of PLN might be the cause of the denervation-induced muscle atrophy (Fig. 1a) because PLN overexpression in mice caused muscle atrophy (Fajardo et al. 2015). However, this hypothesis is not straightforward because PLN fluorescence was found to increase in all fiber types whereas the CSA was reduced only in type IIX and IIB fibers.

Western blotting showed that the expression of SERCA1 was significantly reduced about $\sim 22 \%$ whereas that of SERCA2 was increased by $\sim 3$ times in denervated TA muscles as reported (Dufresne et al. 2016; Schulte et al. 1994). However, the SERCA activity was inhibited by $\sim 40 \%$ after denervation (Fig. 3 and 4). Thus, we assume that the suppression of SERCA activity resulted from the increased expression of PLN. We also found that denervation significantly increased the amount of STIM1 (Fig. 4) (Dufresne et al. 2016). Zhao et al. reported that cardiac STIM1 binds to PLN and thereby enhances SR calcium content (Zhao et al. 2015). Thus, the upregulation of STIM1 from denervation may be compensating for the upregulation of PLN to maintain the SR calcium content after denervation.

We found that the $V_{\max }$ of SERCA activity was significantly decreased, whereas the calcium affinity $\left(\mathrm{pCa}_{50}\right)$ of SERCA was not significantly changed after denervation (Fig. 3). Although a number of previous studies showed that coexpression of PLN with SERCA causes an increase in $\mathrm{pCa}_{50}$ but not a decrease in $V_{\max }$ (Harrer et al. 1995; Slack et al. 1997; Bhupathy et al. 2007), a similar reduction in the $V_{\max }$ of SERCA was observed in the denervated EDL muscles (Dufresne et al. 2016) and in the soleus muscles of a PLN overexpressing mouse (Fajardo et al. 2015; Song et al. 2004). Asahi et al. demonstrated that coexpression of both PLN and SLN, but not coexpression of either alone, with SERCA strongly 
reduced the $V_{\max }$ of SERCA in HEK293 cells (Asahi et al. 2002). Therefore, it is possible that PLN somehow cooperates with endogenous SLN on SERCA in denervated fast muscles.

It is well-known that the profile of protein expression is different between slow- and fast-type muscle fibers (Schiaffino and Reggiani 2011). In general, PLN, SERCA2, and CSQ2 are abundantly expressed in cardiac and slow-type muscles but not in adult fast-twitch muscle. Thus, the increase in these proteins may indicate that denervation causes switching of fast to slow-type fibers (Fig. 4). However, the denervation had a little effect on the fiber distribution of the TA muscle as assessed with antibodies against MHC (Fig. 6a), consistent with previous reports (Huey and Bodine 1998; Raffaello et al. 2006). Furthermore, the significant increase in PLN expression was observed in all analyzed fiber types (Fig. 6b). Thus, it would be an oversimplification to consider that the increase in PLN, SERCA2, and CSQ2 resulted solely from denervation-induced fiber-type switching. Therefore, it is likely that the motor nerve has some direct and specific effect on these proteins in the skeletal muscle.

We showed that the SR calcium content was reduced by half in the denervated muscle (Fig. 2c). However, several studies previously reported that the SR calcium capacity of denervated muscles was increased rather than decreased compared with normal muscles (Brody 1966; Midrio et al. 1997; Germinario et al. 2002). This discrepancy might be due to the different methods adopted to measure SR calcium content. Most previous works quantified the calcium capacity of SR in skinned fibers after loading excess calcium (i.e., the maximum calcium capacity). Conversely, in the present study, we measured the amount of calcium releasable from isolated fibers using ICE treatment in native conditions (i.e., the endogenous calcium capacity). Recently, Murphy et al. demonstrated that the ratio of endogenous to the maximal calcium capacity of SR was not constant among different fiber types (e.g., $22 \%$ in EDL fiber and 69\% in soleus fiber) (Murphy et al. 2009). Therefore, it is also possible that the maximum and endogenous calcium capacity do not move in a parallel fashion in a diseased state. Thus, our and others' findings may not necessarily be contradictory. Our results indicate that operational SR calcium content was significantly reduced after denervation. What is the functional significance of this observation? Previous reports showed that treatment with CPA, a specific SERCA inhibitor, reduced contractile force of isolated muscle fiber of frogs (Même and Léoty 2001) and mice (Divet et al. 2005) in a dose-dependent manner. This indicates that even a partial inhibition of SERCA causes a decrease in operational SR calcium content and contractile force.

We found that denervation induced a decrease in both twitch and tetanus calcium transients but a decrease in only the tetanus contractile force in the TA muscle. Although we do not exactly know the reason for this discrepancy, it is possible that denervation caused an increase in calcium affinity of contractile proteins by promoting expression of slow isoforms of troponins (Leeuw et al. 1994; Rana et al. 2005).

It is previously established that a reduction of SERCA2 activity triggers a decrease in the SR calcium content and contractility of failing cardiac myocytes, as reviewed by (Kranias and Hajjar 2012). It has also been shown that suppression of PLN function has therapeutic effects on heart failure (Schmidt et al. 2001; Kranias and Hajjar 2012; Kho et al. 2012). Thus, it is possible that a PLN-targeting therapy is 
1 developed for denervation-induced skeletal muscle diseases including age-dependent sarcopenia in the 2 future. 
We are grateful to Ms. Reiko Sakai for her secretarial assistance. This work was supported by 4 Grants-in-aid for Scientific Research 25670641 (to HK) and 16K20046 (to MK) from the Ministry of 5 Education, Culture, Sport, Science and Technology of Japan (MEXT) and by a research grant 41290769 6 from MSDKK (Tokyo, Japan) to MY. 
3 Allard B (2018) From excitation to intracellular $\mathrm{Ca}^{2+}$ movements in skeletal muscle: Basic aspects and related clinical disorders. Neuromuscul Disord 28:394-401.

Asahi M, Kurzydlowski K, Tada M, MacLennan DH (2002) Sarcolipin inhibits polymerization of phospholamban to induce superinhibition of sarco(endo)plasmic reticulum $\mathrm{Ca}^{2+}$-ATPases (SERCAs). J Biol Chem 277:26725-26728.

Bhupathy P, Babu GJ, Periasamy M (2007) Sarcolipin and phospholamban as regulators of cardiac sarcoplasmic reticulum $\mathrm{Ca}^{2+}$ ATPase. J Mol Cell Cardiol 42:903-911.

Bloemberg D, Quadrilatero J (2012) Rapid determination of myosin heavy chain expression in rat, mouse, and human skeletal muscle using multicolor immunofluorescence analysis. PLoS ONE 7:e35273.

Brini M, Carafoli E (2009) Calcium pumps in health and disease. Physiol Rev 89:1341-1378.

Brody IA (1966) Relaxing factor in denervated muscle: A possible explanation for fibrillations. Am J Physiol 211:1277-1280.

Cosgrove BD, Gilbert PM, Porpiglia E, et al (2014). Rejuvenation of the muscle stem cell population restores strength to injured aged muscles. Nat. Med. 20:255-264.

Divet A, Lompré A-M, Huchet-Cadiou C (2005) Effect of cyclopiazonic acid, an inhibitor of the sarcoplasmic reticulum Ca-ATPase, on skeletal muscles from normal and mdx mice. Acta Physiol Scand 184:173-186.

Dufresne SS, Dumont NA, Boulanger-Piette A, et al (2016) Muscle RANK is a key regulator of $\mathrm{Ca}^{2+}$ storage, SERCA activity, and function of fast-twitch skeletal muscles. Am J Physiol Cell Physiol 310:C663-72.

Duhamel TA, Green HJ, Stewart RD, et al (2007) Muscle metabolic, SR $\mathrm{Ca}^{2+}$-cycling responses to prolonged cycling, with and without glucose supplementation. J Appl Physiol 103:1986-1998.

Fajardo VA, Bombardier E, McMillan E, et al (2015) Phospholamban overexpression in mice causes a centronuclear myopathy-like phenotype. Dis Model Mech 8:999-1009. and sarcolipin in human skeletal muscle fibers. PLoS ONE 8:e84304. rat EDL skeletal muscle. J Appl Physiol 92:2045-2052. 
1 Gonzalez-Freire M, de Cabo R, Studenski SA, Ferrucci L (2014) The neuromuscular junction: Aging at the crossroad between nerves and muscle. Front Aging Neurosci 6:208.

3 Harrer JM, Ponniah S, Ferguson DG, Kranias EG (1995) Expression of phospholamban in C2C12 cells and regulation of endogenous SERCA1 activity. Mol Cell Biochem 146:13-21.

Huey KA, Bodine SC (1998) Changes in myosin mRNA and protein expression in denervated rat soleus and tibialis anterior. Eur J Biochem 256:45-50.

Jorgensen AO, Jones LR (1986) Localization of phospholamban in slow but not fast canine skeletal muscle fibers. An immunocytochemical and biochemical study. J Biol Chem 261:3775-3781.

Kho C, Lee A, Hajjar RJ (2012) Altered sarcoplasmic reticulum calcium cycling--targets for heart failure

Kranias EG, Hajjar RJ (2012) Modulation of cardiac contractility by the phospholamban/SERCA2a regulatome. Circ Res 110:1646-1660.

Leeuw T, Kapp M, Pette D (1994) Role of innervation for development and maintenance of troponin subunit isoform patterns in fast- and slow-twitch muscles of the rabbit. Differentiation 55:193-201.

Loirat MJ, Lucas-Heron B, Ollivier B, Léoty C (1988) Calcium binding protein changes of sarcoplasmic reticulum from rat denervated skeletal muscle. Biosci Rep 8:369-378.

Loy RE, Orynbayev M, Xu L et al (2011) Muscle weakness in Ryr1I4895T/WT knock-in mice as a result of reduced ryanodine receptor $\mathrm{Ca}^{2+}$ ion permeation and release from the sarcoplasmic reticulum. $\mathrm{J}$ Gen Physiol 137:43-57.

Même W, Léoty C (2001) Cyclopiazonic acid and thapsigargin reduce $\mathrm{Ca}^{2+}$ influx in frog skeletal muscle fibres as a result of $\mathrm{Ca}^{2+}$ store depletion. Acta Physiol Scand 173:391-399.

Midrio M (2006) The denervated muscle: Facts and hypotheses. A historical review. Eur J Appl Physiol 98:1-21.

Midrio M, Danieli-Betto D, Megighian A, Betto R (1997) Early effects of denervation on sarcoplasmic reticulum properties of slow-twitch rat muscle fibres. Pflugers Arch 434:398-405. and maximal $\mathrm{Ca}^{2+}$ storage levels in sarcoplasmic reticulum of fast- and slow-twitch fibres of rat. $\mathrm{J}$ 
Nakagawa T, Yokoe S, Asahi M (2016) Phospholamban degradation is induced by phosphorylation-mediated ubiquitination and inhibited by interaction with cardiac type Sarco(endo)plasmic reticulum $\mathrm{Ca}^{2+}$-ATPase. Biochem Biophys Res Commun 472:523-530.

Palexas GN, Savage N, Isaacs H (1981) Characteristics of sarcoplasmic reticulum from normal and denervated rat skeletal muscle. Biochem J 200:11-15.

Park KHJ (2015) Mechanisms of muscle denervation in aging: Insights from a mouse model of amyotrophic lateral sclerosis. Aging Dis 6:380-389.

Periasamy M, Kalyanasundaram A (2007) SERCA pump isoforms: their role in calcium transport and disease. Muscle Nerve 35:430-442.

Péréon Y, Sorrentino V, Dettbarn C et al (1997) Dihydropyridine receptor and ryanodine receptor gene expression in long-term denervated rat muscles. Biochem Biophys Res Commun 240:612-617.

Raffaello A, Laveder P, Romualdi C et al (2006) Denervation in murine fast-twitch muscle: short-term physiological changes and temporal expression profiling. Physiol Genomics 25:60-74.

Rana ZA, Gundersen K, Buonanno A et al (2005) Imaging transcription in vivo: distinct regulatory effects of fast and slow activity patterns on promoter elements from vertebrate troponin I isoform genes. J Physiol 562:815-828

Rudolf R, Khan MM, Labeit S, Deschenes MR (2014) Degeneration of neuromuscular junction in age and dystrophy. Front Aging Neurosci 6:99.

Salvatori S, Damiani E, Zorzato F et al (1988) Denervation-induced proliferative changes of triads in rabbit skeletal muscle. Muscle Nerve 11:1246-1259.

Schiaffino S, Reggiani C (2011) Fiber types in mammalian skeletal muscles. Physiol Rev 91:1447-1531.

Schmidt AG, Edes I, Kranias EG (2001) Phospholamban: a promising therapeutic target in heart failure? Cardiovasc Drugs Ther 15:387-396.

Schulte L, Peters D, Taylor J, et al (1994) Sarcoplasmic reticulum $\mathrm{Ca}^{2+}$ pump expression in denervated skeletal muscle. Am J Physiol 267:C617-22. 
1 Song Q, Young KB, Chu G et al (2004) Overexpression of phospholamban in slow-twitch skeletal muscle is associated with depressed contractile function and muscle remodeling. FASEB J 18:974-976

3 Teng ACT, Miyake T, Yokoe S et al (2015) Metformin increases degradation of phospholamban via 4 autophagy in cardiomyocytes. Proc Natl Acad Sci USA 112:7165-7170.

5 Yokoe S, Asahi M (2017) Phospholamban Is Downregulated by pVHL-Mediated Degradation through $6 \quad$ Oxidative Stress in Failing Heart. Int J Mol Sci 18:2232.

7 Zhao G, Li T, Brochet DXP, et al (2015) STIM1 enhances SR $\mathrm{Ca}^{2+}$ content through binding 8 phospholamban in rat ventricular myocytes. Proc Natl Acad Sci USA 112:E4792-801. 
3 Fig. 1 Changes in the physical properties and contractile force of the mouse TA muscle after denervation.

4 a. A decrease in muscle mass and cross-sectional area after denervation. The TA muscle was isolated

5 from control (Cont) and denervated (DEN) hindlimb on the $14^{\text {th }}$ day of the sciatic nerve denervation $(n=$

6 12). ${ }^{* *} P<0.01$ compared with Cont. b. A decrease in contractile force of the TA muscle after

7 denervation. Top panels: representative traces of the contractile force of twitch and tetanus. Bottom

8 panels: absolute force of twitch and tetanus $(\mathrm{n}=12) . * * P<0.01$ compared with Cont. c. contractile

9 properties of the TA muscle after denervation. Top panels: specific force of twitch and tetanus $(\mathrm{n}=12)$.

10 Middle panels: the maximum contraction rate $(+\mathrm{dF} / \mathrm{dT})$ of twitch and tetanus. Bottom panels: the

11 maximum relaxation rate $(-\mathrm{dF} / \mathrm{dt})$ of twitch and tetanus. $* P<0.05$ and $* * P<0.01$ compared with Cont

13 Fig. 2 Changes in calcium transient of the FDB muscle after denervation. Calcium imaging of isolated

14 FDB fibers was performed with a fluorescence calcium indicator Fluo-4. Calcium transients were elicited 15 by twitch-, tetanic-, or ICE- (ionomycin, CPA, and EGTA) stimulations. a. Upper panels show

16 representative traces and peak amplitude of the calcium transients elicited by twitch stimulation. Bottom

17 panels show the time to peak and half decay time (T1/2) of the calcium transient elicited by twitch

18 stimulation, respectively $(\mathrm{n}=48-41)$. ${ }^{* *} P<0.01$ compared with Cont. $\mathbf{b}$. Representative traces and peak

19 amplitude of the calcium transients elicited by tetanic stimulation $(\mathrm{n}=43-52)$. $* * P<0.01$ compared with

20 Cont. c. Representative traces and peak amplitude of the calcium transients elicited by ICE stimulation (n

$21=43-52) . * * P<0.01$ compared with Cont

Fig. 3 SERCA activity of the TA muscle. a. Relationship between SERCA activity and calcium concentration. Microsomal proteins were isolated from control (Cont) and denervated (DEN) TA muscles on the $14^{\text {th }}$ day of denervation. The CPA-sensitive calcium ATPase activity of microsomes was quantified as inorganic phosphate production (symbols and bars) $(n=4)$. Lines indicating the fitting of data into the Hill equation (Eq. 1). b. The graph showing $\mathrm{pCa}_{50}$ and $V_{\max }$ estimated from curve fitting (A). $* P<0.05$ compared with Cont

Fig. 4 Changes in the expression of proteins involved in the excitation-contraction (EC) coupling of the skeletal muscle and mRNA of PLN. a. Representative image of the western blotting of proteins involved in EC coupling. Microsome proteins were isolated from control (Cont) and denervated (DEN) TA muscles on the $14^{\text {th }}$ day of sciatic nerve denervation. The same amount of proteins were assayed with western blotting with indicated antibodies. $\mathbf{b}$. Quantification of protein expressions. The protein expressions in the TA muscles assessed by western blotting were quantified by ImageJ. The data were normalized to Cont (SERCA1 and SERCA2, $n=8$; other proteins, $n=4$ ). c. Expressions of PLN in TA muscles. Microsome proteins were isolated from control (Cont) and denervated (DEN) TA muscles on the $14^{\text {th }}$ day of sciatic nerve denervation. The expression of PLN was assessed with two different 
1 antibodies (mouse monoclonal (M-mono) and rabbit polyclonal (R-poly) antibodies). The PLN

2 expression as assessed by western blotting was quantified by ImageJ. The data were normalized to Cont

$3(\mathrm{n}=4) * * P<0.01$ compared with contralateral muscles. d. Expression of PLN mRNA in control (Cont)

4 and denervated (DEN) muscles. The amount of PLN mRNA in TA muscles was quantified with qPCR.

5 The data were first normalized to the amount of GAPDH and then to Cont $(\mathrm{n}=5)$

7 Fig. 5 Changes in the PLN expression in soleus muscles after denervation. a. Expressions of PLN in the

8 soleus muscles. Microsome proteins were isolated from control (Cont) and denervated (DEN) soleus

9 muscles on the $14^{\text {th }}$ day of sciatic nerve denervation. The PLN expression as assessed by western blotting

10 was quantified by ImageJ. The data were normalized to Cont $(\mathrm{n}=4)$. ${ }^{* *} P<0.01$ compared with

11 contralateral muscles. b. Expression of PLN mRNA in control (Cont) and denervated (DEN) muscles.

12 The amount of PLN mRNA in soleus muscles was quantified with qPCR. The data were first normalized

13 to the amount of GAPDH and then to Cont $(\mathrm{n}=5)$

15 Fig. 6 Immunohistochemical analysis of PLN expression. a. Fiber populations and cross-sectional areas 16 of the TA muscle. TA muscles were isolated from control (Cont) and denervated (DEN) hindlimbs on the $1714^{\text {th }}$ day of sciatic nerve denervation. The TA muscle was separated into red (RTA) and white (WTA)

18 portions. Left-hand panel shows the fiber type distribution identified with immunohistochemical analysis 19 of MHC expression. The percentage of each fiber was calculated from immunohistochemical images 20 (>331 fibers were counted in each image derived from four animals). $* P<0.05$ compared with contralateral muscles. Cross-sectional areas of the fiber were also measured from the immunohistochemical images of RTA (cross-sectional area of $>30$ fibers of each type derived from three animals were calculated). b. A change in PLN expression in each fiber type of the TA muscle after denervation. A left-hand panel: representative immunohistochemical images of MHC and PLN. Bar $=50$ $\mu \mathrm{m}$ scale. A right-hand panel: fluorescence intensity of PLN of each fiber type determined from immunohistochemical images of RTA (PLN intensity in $>30$ fibers of each type derived from three animals were calculated). $* * P<0.01$ compared with contralateral muscles 


\section{Fig. 1}

A

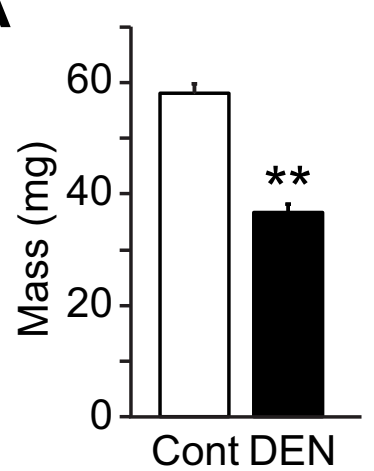

B Twitch
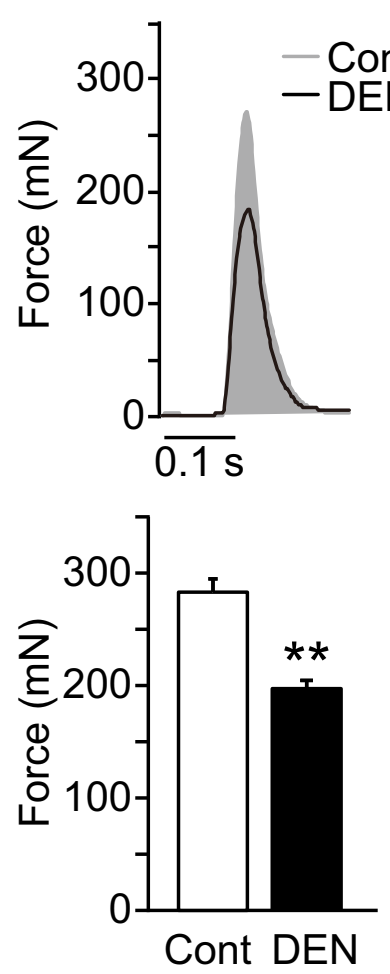

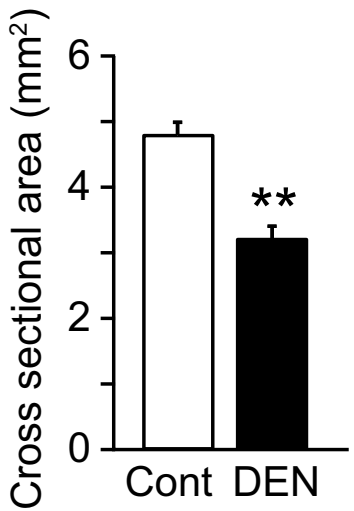

C
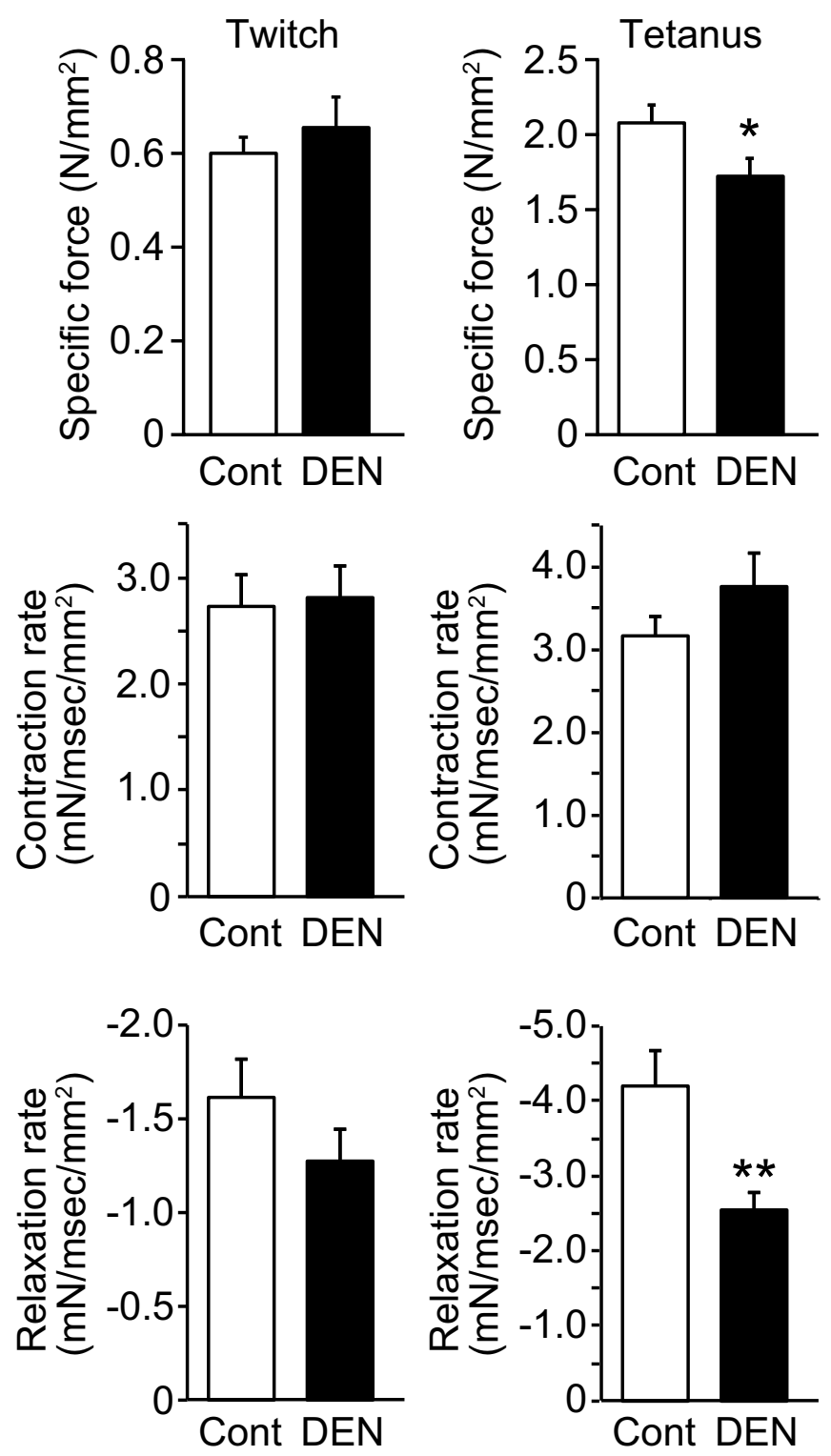


\section{Fig. 2}

A

Twitch
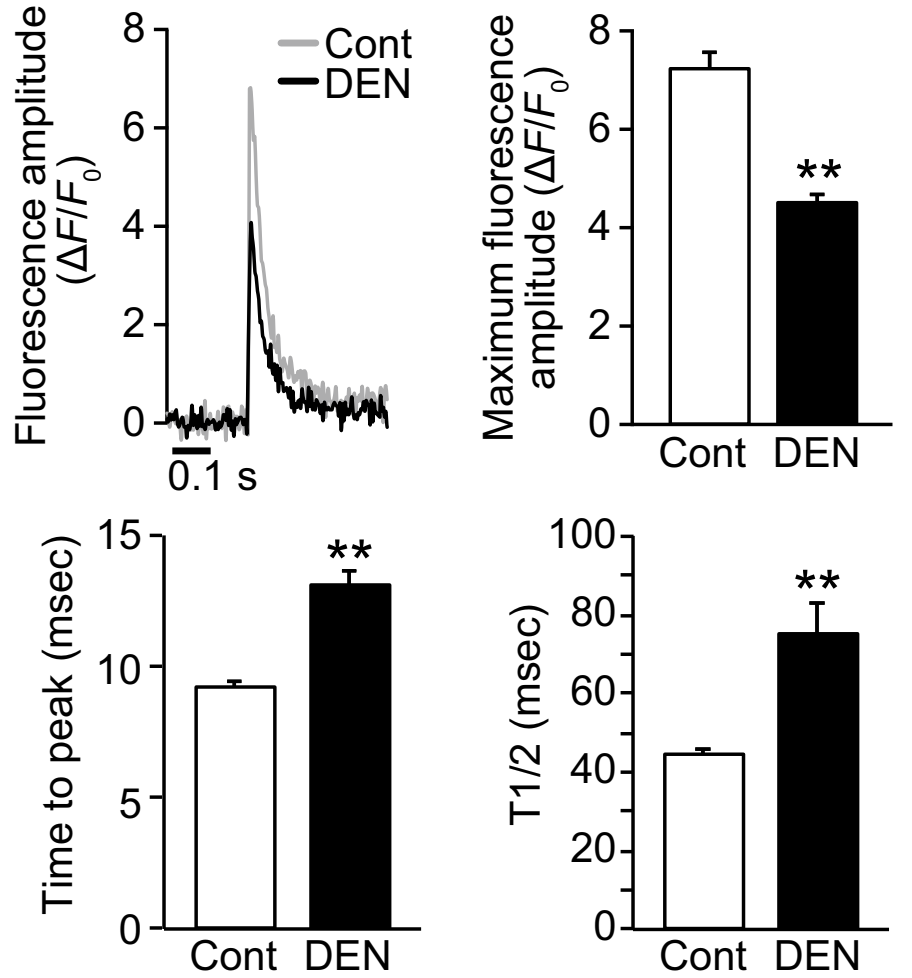

B

Tetanus

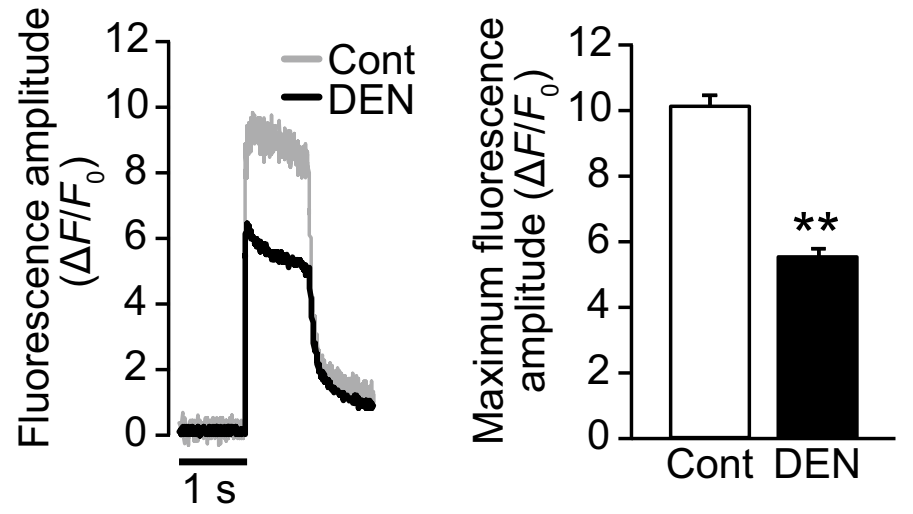

C

ICE

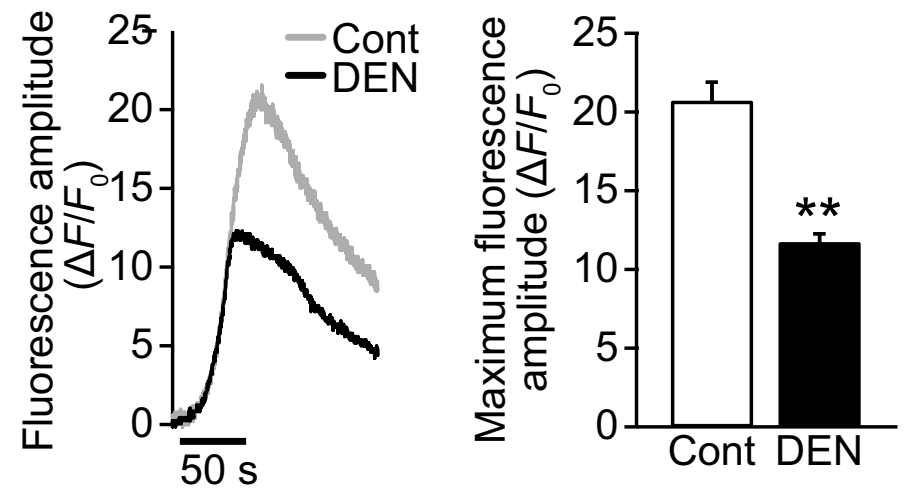


A

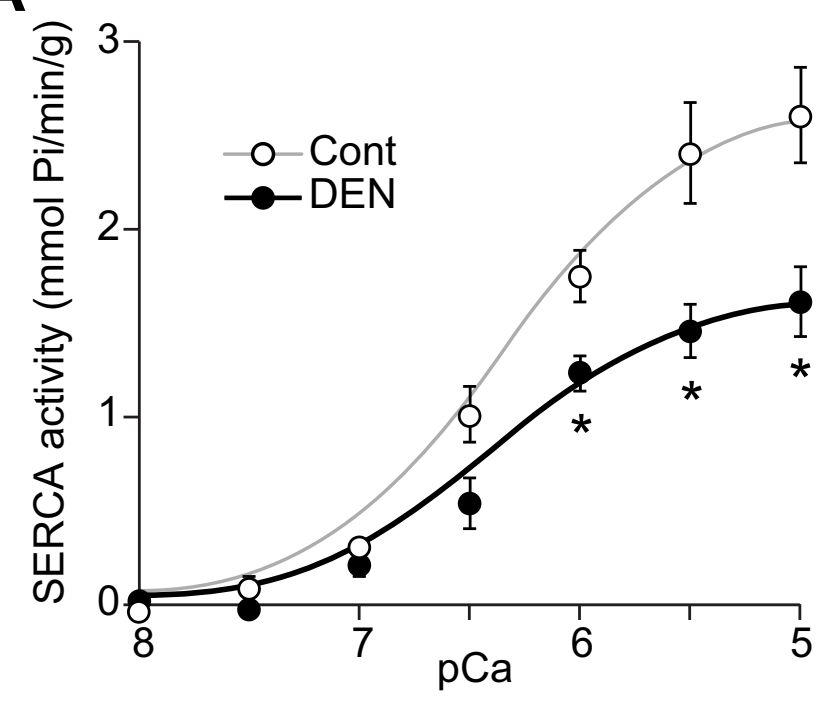

B

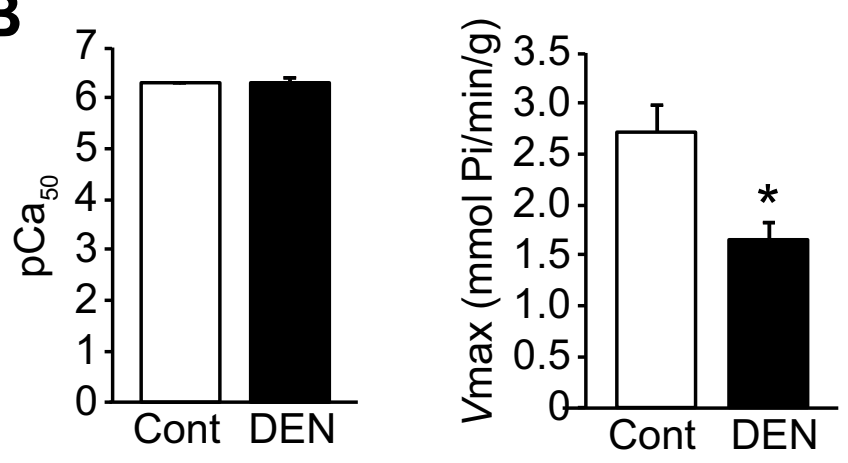


A B

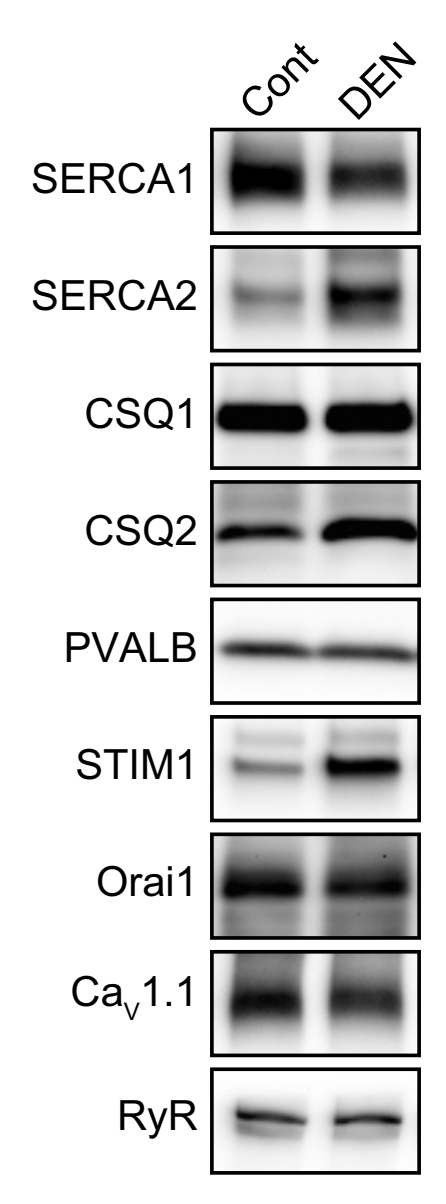

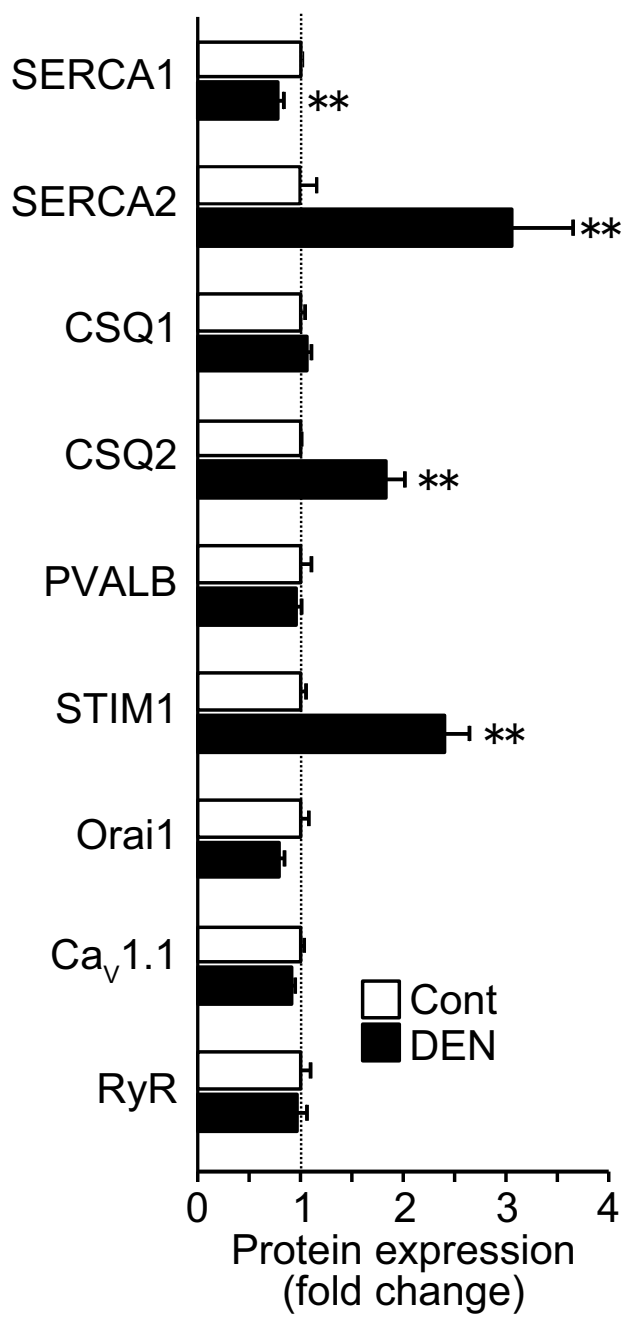

C
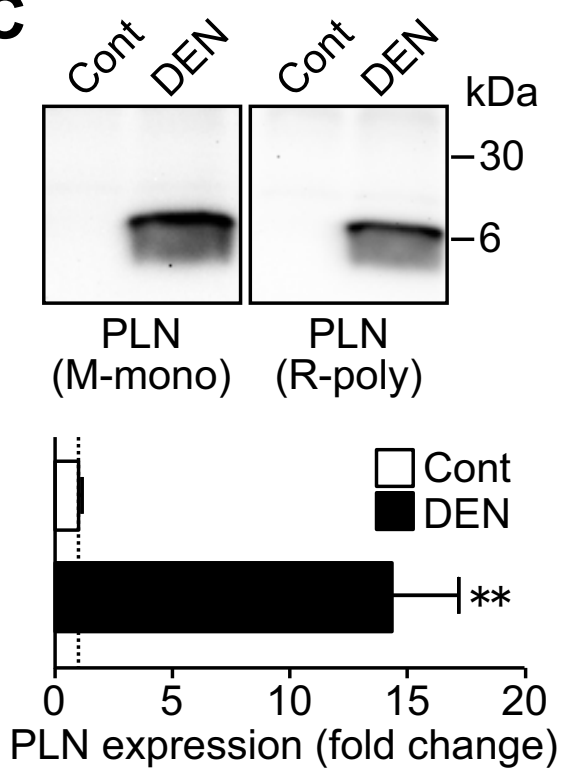

D

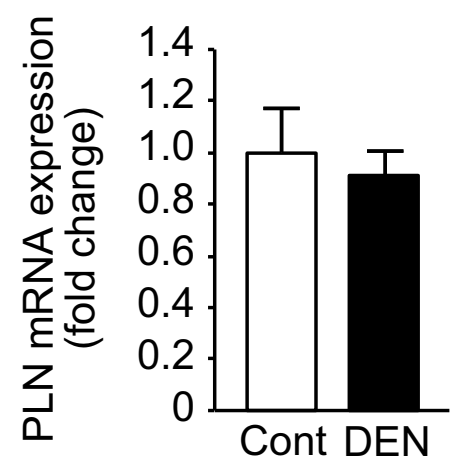


Fig. 5

A
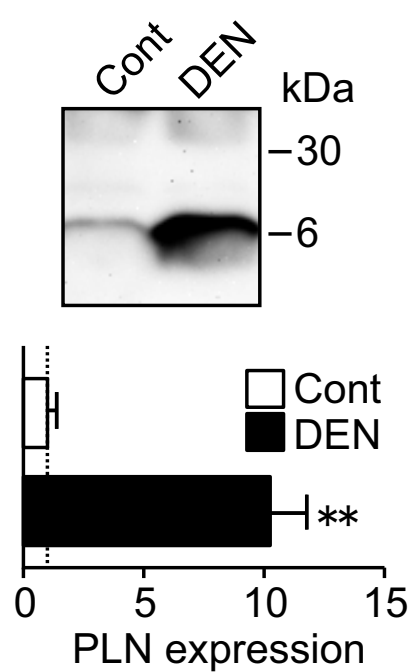

(fold change)

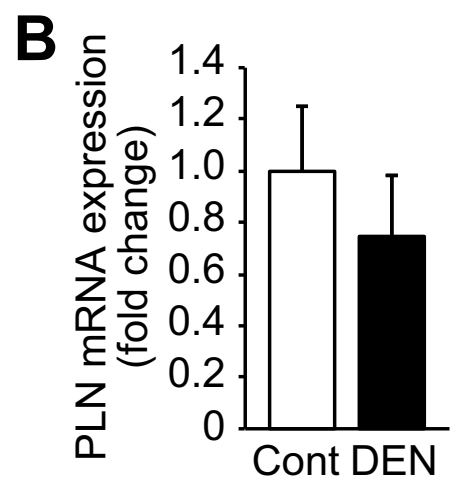




\section{Fig. 6}

A
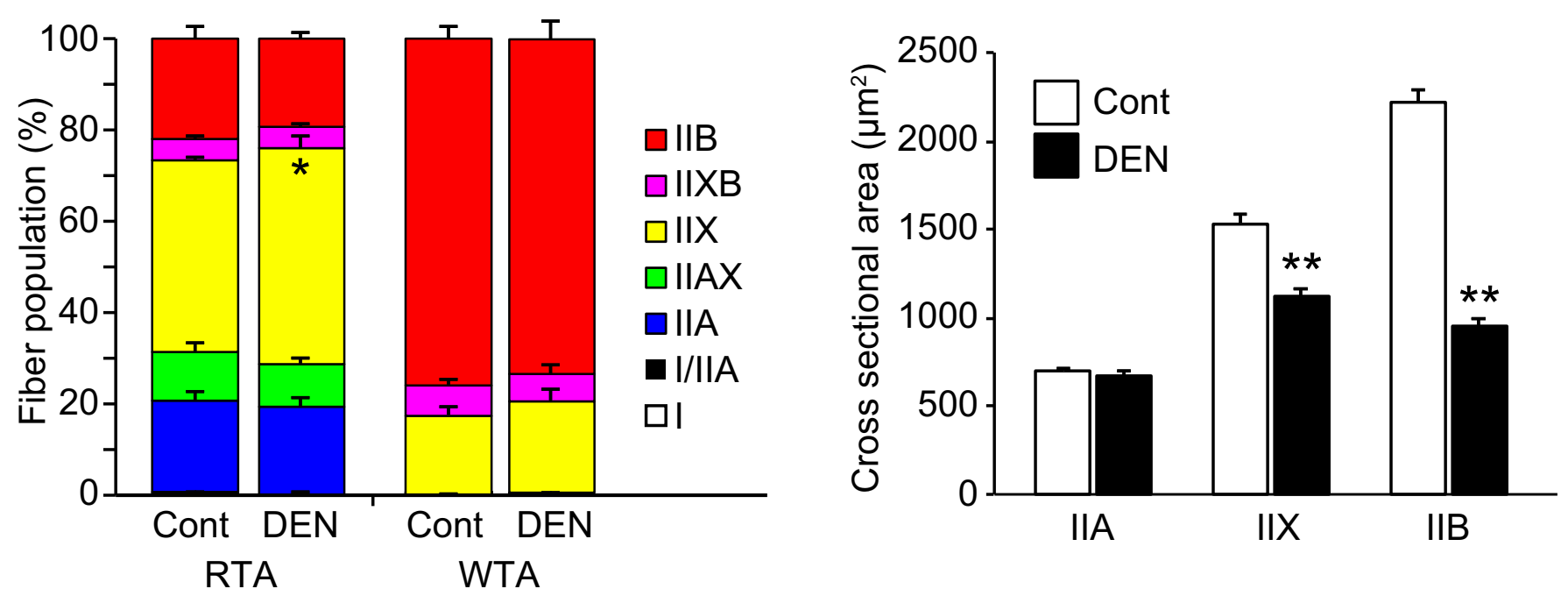

B
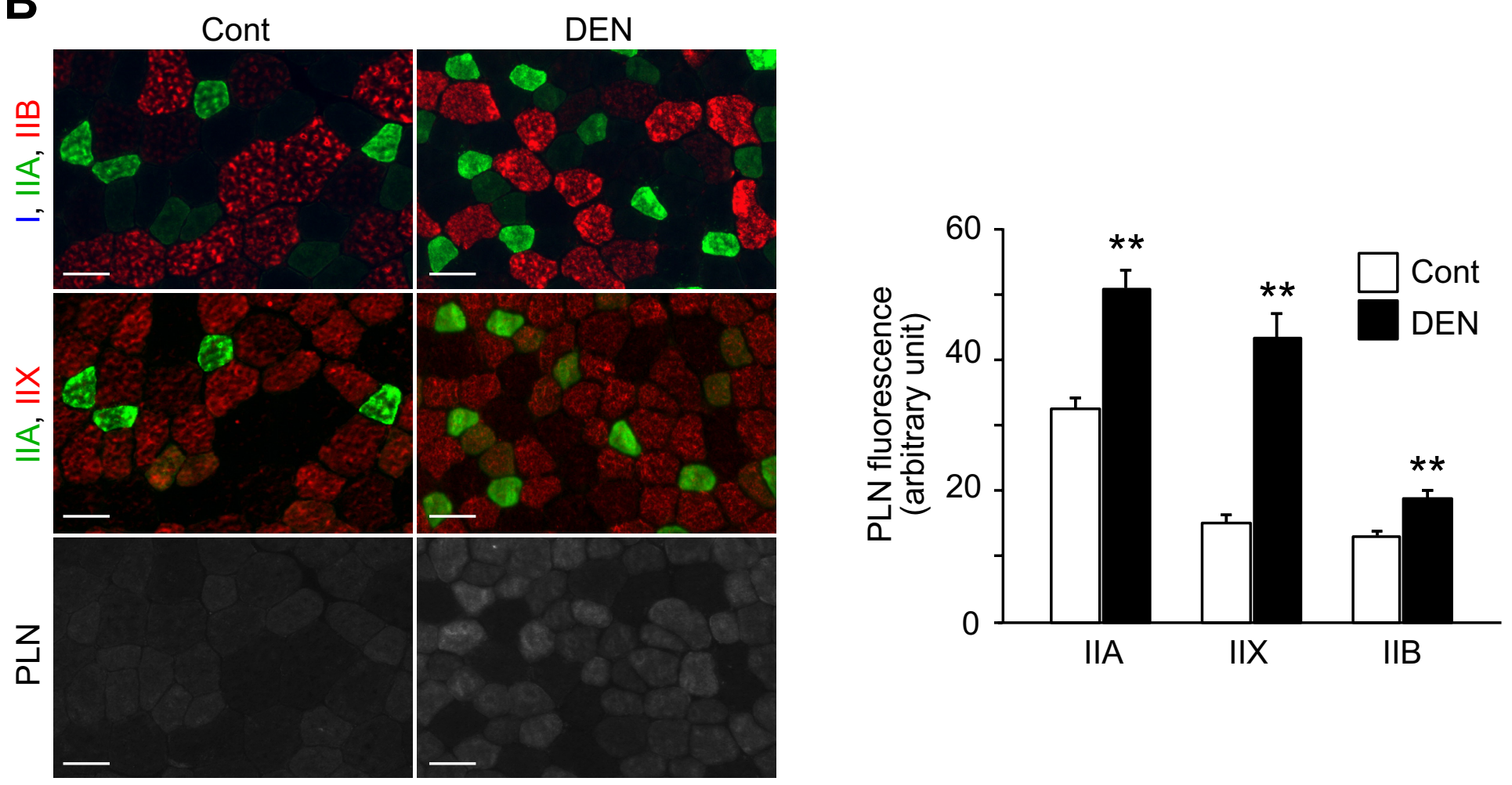